\title{
Recent Advances in the Management of Stage IV Colon Cancer
}

\author{
Rani Kanthan ${ }^{1}$, Jenna-Lynn Senger ${ }^{1}$, Shahid Ahmed ${ }^{2}$, Selliah Kanthan ${ }^{3}$ \\ ${ }^{1}$ Department of Pathology and Laboratory Medicine, University of Saskatchewan, Saskatoon, Canada; ${ }^{2}$ Medical Oncologist, Saska- \\ toon Cancer Centre, Saskatoon, Canada; ${ }^{3}$ Department of Surgery, University of Saskatchewan, Saskatoon, Canada. \\ Email: rani.kanthan@saskatoonhealthregion.ca
}

Received October $20^{\text {th }}, 2012$; revised November $22^{\text {nd }}, 2012$; accepted December $2^{\text {nd }}, 2012$

\begin{abstract}
Colon cancer is the second commonest cause of cancer-related death in Canadian men and women, with approximately one-third of patients dying from this disease. One quarter of patients present with metastases initially, and up to half of all colon cancer patients will develop stage IV disease over the course of their life. Despite ongoing advances in the evolution of newer cytotoxic drugs, targeted biological agents and improved metastasectomy techniques, the gain in overall survival in these patients is of low magnitude. This manuscript is a targeted review of the recent advances over the last decade in the management of advanced stage IV colon cancer as available in the published English literature. The two major arms of metastatic colon cancer management that include surgery and systemic chemotherapy and palliative measures as available are discussed. A multi-modality team-based approach involving medical oncologists, surgical oncologists, radiologists, and other health-care providers continues to be critical for ongoing success in the therapeutic management of these patients. Future studies of well-designed prospective, randomized-controlled clinical trials to develop and evaluate newer therapeutic strategies are recommended for continued and improved understanding for optimization of clinical management in advanced colon cancer.
\end{abstract}

Keywords: Stage IV Colon Cancer; Systemic Therapy; Surgical Management

\section{Introduction}

Despite ongoing advancements in screening and early detection such as fecal molecular markers [1], approximately one million new cases of colon cancer continue to be diagnosed annually with 500,000 deaths being directly attributed to the same each year [2]. Amongst these new cases, $19 \%-25 \%$ present initially with metastatic disease. Additionally, up to $50 \%$ of patients with early stage disease will develop metastases during their lifetime [2-4]. Though early stage colon cancer is associated with a 5 -year survival rate of $74 \%$, this percentage declines dramatically to $5 \%-10 \%$ in stage IV disease [5]. Stage IV colon cancer is characterized by metastatic spread to other organs via the blood and the lymphatics. The involvement of a single extra-colonic organ is designated as stage IVA and metastases in multiple organs as stage IVB. The most common site of extra-colonic metastases is the liver followed by the lung and the peritoneum. The genetic and epigenetic changes associated with this metastatic spread remain poorly understood. Recent advances in molecular studies, however, indicate that multiple markers such as growth factors (prostaglandin E2, epithelial growth factor, and vascular endothelial growth factor) and the epithelial-mesenchymal transition mechanisms may be involved [6].

As pharmacological, radiotherapeutic, and surgical fields diversify and progress; management strategies to treat advanced colon cancer are continually evolving. Currently, the majority of patients with stage IV disease are treated with chemotherapy and biological agents; however, no consensus guidelines are universally accepted for the best-practice management of stage IV colon cancer. Treatment standardization is additionally hindered by two competing factors: 1) patient population with different expectations and goals, and 2) tumor profiles with varied chemotherapeutic sensitivity and resectability. Although for most patients with advanced colon cancer (CC), the treatment goals are to prolong overall survival (OS) and to maintain quality of life (QOL) for as long as possible, some patients with advanced CC particularly those with limited metastases can be cured following targeted metastasectomy [7,8] (Figure 1). Of note, selected patients with initially unresectable disease may become eligible for resection if they achieve a good response to systemic therapy. $75 \%-90 \%$ of stage IV colon cancers are not suitable for curative 


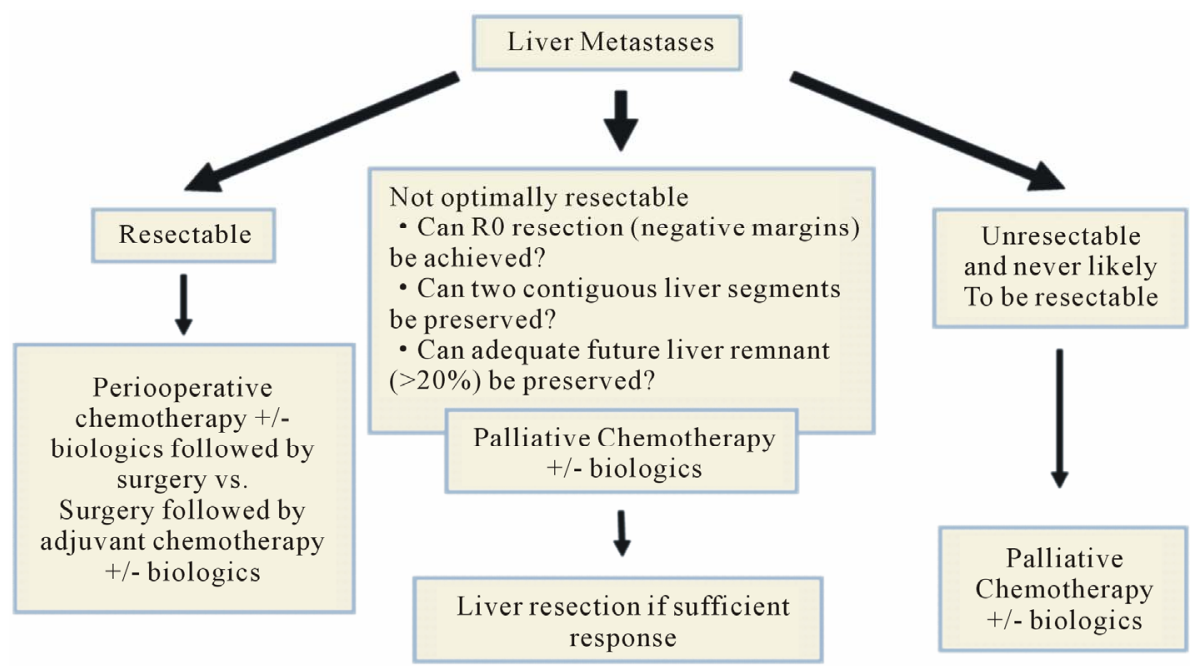

Figure 1. Treatment choice of patients with advanced CC and liver metastases.

resection [9] due to tumor-specific (inextirpable primary, multiple metastases) or patient-specific (age, co-morbidities) reasons [10]. These patients may benefit from systemic and local therapy [11].

The median overall survival of patients with advanced $\mathrm{CC}$ with supportive care alone is only about $5-6$ months [12]. Systemic therapy provides meaningful improvements in both progression-free and overall survival. For many years, 5-fluorouracil (5-FU) was the only active agent available in the management of patients with advanced CC $[13,14]$. Over the past decade access to several novel agents including the cytotoxic agents irinotecan, oxaliplatin and humanized monoclonal antibodies: 1) bevacizumab, 2) cetuximab and 3) pani-tumumab that target vascular endothelial growth factor (VEGF) and epidermal growth factor receptors (EGFR) respecttively have resulted in significant improvement in the survival of patients with advanced CC [15-28]. More recently regorafenib, an orally active inhibitor of angiogenic/stromal/oncogenic receptor tyrosine kinases, and aflibercet a recombinant fusion protein that interferes with VEGF binding have also shown survival benefit in previously treated unresponsive patients $[29,30]$. With the judicious use of active agents in the management of advanced CC, the median overall survival of patients has increased to 2 years. Selection of appropriate therapy may be complicated as many patients are willing to accept a short survival prolongation despite considerable toxicity [10]. These factors indicate the need for an individualized approach in management, involving a multidisciplinary team including colorectal/hepatobiliary/thoracic surgeons, medical/radiation oncologists, pathologists, interventional radiologists, nurses, and psychosocial support services [31]. In this manuscript, we review the recent advances in the therapeutic options available for the management of stage IV colon cancer.

\section{Materials and Methods}

A review of the published English literature was conducted using the search engines PubMed, Medline, and Google Scholar. The search terms ["Stage IV colorectal cancer" OR "advanced colorectal cancer" OR "advanced colon cancer"] were combined with the search terms ["systemic therapy" OR "chemotherapy" OR "targeted therapy" OR "anti-EGFR therapy" OR "anti-VEGF therapy" OR "conversion therapy" OR "neoadjuvant therapy" OR "surgery" OR "metastasectomy" OR "stent" OR "resection" OR "treatment" OR "therapy"]. Selection criteria included articles limited to the past five years, focusing on stage IV colon cancer and recent advances in management strategies. Articles were analyzed and appropriate secondary references procured as needed. A total of approximately 200 articles were retrieved and read, of which 98 were selected for reference in this review.

The following sections will focus discussion on the two major components of advanced colon cancer management that includes systemic therapy (part 1), surgery (part 2) and palliative measures as available.

\section{Part 1: Systemic Therapy}

The introduction of 5-fluorouracil (5-FU), a fluropyrimidine has increased the median overall survival of patients with advanced CC to approximately $10-12$ months. For many decades, 5-FU remained the single active agent that was used in the management of advanced CC. In the late 1980s, the addition of biomodulator leucovorin $(\mathrm{LV})$ to 5 -FU resulted in improved response rate with increased overall survival [32]. A decade later both irinotecan and oxaliplatin demonstrated efficacy as second-line treatments, and subsequently have been approved as first-line therapies for advanced 
$\mathrm{CC}$ with improvement in median overall survival to 15 20 months [15-18,33]. Biological therapies such as cetuximab and bevacizumab improve average survival to approximately 20 - 24 months [22-27]. Despite the availability of newer agents, fluoropyrimidines continue to form the backbone of systemic therapy in the management of advanced CC. The following section will review the evolving trends in systemic therapy for the management of stage IV CC over the past two decades.

\subsection{Fluropyrimidines}

\subsubsection{5-FU/Leucovorin}

Before the advent of combination regimens, 5-FU/LV was the standard first-line therapy for advanced CC and continues to be used in patients who cannot tolerate combination chemotherapy. Several doses and schedules of 5-FU and LV are in clinical use. The two widely used bolus regimens are the Mayo and Roswell Park regimens $[34,35]$. In a randomized trial short-term infusional 5-FU/ LV compared with bolus 5FU/LV was associated with 1) significantly better response rate (33 versus $14 \%$ ); 2 ) improved median progression free survival (PFS) [28 vs 22 weeks]; and 3) a trend toward longer overall survival (OS) (62 vs 57 weeks) [36].

\subsubsection{Capecitabine}

Capecitabine is an orally active fluoropyrimidine that is converted to 5-FU in three sequential enzymatic reactions. Two randomized phase III trials comparing capecitabine versus bolus 5-FU/LV (Mayo Clinic regimen) demonstrated similar PFS and OS with less toxicity [37, 38].

\subsection{Irinotecan}

Irinotecan as a single agent in patients with advanced CC for whom 5-FU-LV has failed, has demonstrated 2 - 3 months survival advantage [39]. Three key phase III trials demonstrated a survival benefit when irinotecan was combined with 5-FU/LV compared to 5-FU/LV alone in chemotherapy naïve patients $[16,40,41]$. In contrast to the European trials where infusional 5-FU was used, in the American study irinotecan was evaluated in combination with bolus 5-FU/LV (IFL) [41]. Median survival varied from 17.4 - 20.1 months with irinotecan in combination with infusional 5FU (FOLFIRI) compared with 14.1 - 16.9 months with infusional 5FU alone $[15,40]$. Due to relatively higher toxicity and lower efficacy, bolus 5-FU-based irinotecan combinations such as IFL is no longer considered to be an appropriate choice for irinotecan/5-FU/LV therapy. Irinotecan has also been explored in combination with capecitabine (XELIRI, CAPIRI). However compared with FOLFIRI combination of capecitabine/irinotecan has been associated with significantly higher rates of toxicity, inferior PFS (5.8 versus 7.6 months), and a trend towards inferior median survival (18.9 versus 23.1 months) [23].

\subsection{Oxaliplatin}

Oxaliplatin as a single agent has limited value, and is most efficacious when clinically combined with infusional 5-FU/LV in the FOLFOX regimen for patients with advanced colon cancer [42]. At least three trials show significantly greater response rates in PFS with oxaliplatin plus short-term infusional 5-FU/LV (FOLFOX) compared to 5-FU/LV alone in the first-line setting [17, 43,44]. The efficacy and tolerability of oxaliplatin in combination with capecitabine (XELOX or CAPOX) has been explored extensively as both first and second-line therapy $[45,46]$. A systematic review of trials comparing first-line CAPOX versus FOLFOX concluded that CAPOX was associated with a significantly lower response rate, but this did not translate into lower PFS or OS [46].

\subsection{FOLFOX versus FOLFIRI as First-Line Therapy}

A phase III trial involving 220 patients compared first line FOLFOX6 with FOLFIRI. On progression of the disease, both groups were allowed crossover. Overall, no significant difference was noted with respect to response rate, PFS, and OS with approximately 21 months survival in both groups [18]. A second phase III study of 336 patients randomized to FOLFOX4 versus FOLFIRI subsequently confirmed this result [19]. As a result, FOLFOX and FOLFIRI are both considered acceptable first-line therapy for patients with advanced CC.

\section{Oxaliplatin plus Irinotecan Alone or with 5-FU/LV}

Combination of irinotecan and oxaliplatin with and without 5-FU/LV has been studied, both as first and second-line therapy. In an Intergroup trial, the oxaliplatin and irinotecan combination (IROX) regimen was found to be inferior to first-line FOLFOX4. It was also more toxic in elderly individuals [47].

Two small randomized trials evaluated the efficacy of all three active drug combinations (infusional 5FU, irinotecan, and oxaliplatin, FOLFOXIRI) as first-line therapy in patients with advanced CC. An Italian trial of 244 chemotherapy naïve patients suggested superiority of FOLFOXIRI over FOLFIRI. FOLFOXIRI was associated with significantly higher response rate (66 versus $41 \%$ ), better overall survival (23.4 versus 16.7 months) and higher number of liver metastasectomy [21,48]. However, a Hellenic Oncology group trial of 283 patients with previously untreated advanced CC noted no significant benefit for three-drug combinations (FOLFOXIRI) over FOLFIRI [49]. Moreover, relative efficacy of 
FOLFOXIRI versus FOLFOX/CAPOX or FOLFIRI in combination with a biologic such as bevacizumab or cetuximab remains unknown.

\subsection{Sequential versus Combined Therapy}

Fluoropyrimidines, irinotecan, and oxaliplatin are the three approved conventional cytotoxic agents that have demonstrated efficacy in advanced CC. Oxaliplatin does not have single-agent activity and is therefore best used in combination with fluropyrimidines. Exposure to all three drug classes during the course of treatment has been associated with a better survival [50].

Highest survival figures have been obtained in studies where first-line treatment with doublets or triplets have been administered. In the CAIRO 1 and the MRC FOCUS trials patients were randomized between sequential and upfront combination chemotherapy [51,52]. Although the authors concluded that sequential single agent therapy did not compromise overall survival, median survival in all groups was lower than the usual median OS of 20 months or longer obtained in combination therapy trials. While this difference in survival could partly be explained by differences in patient characteristics, the general opinion is that combination therapy should be the standard and sequential treatment should be limited to selected cases [50]. The well-established combination doublets are FOLFOX (5FU, leucovorin, and oxaliplatin), XELOX (capecitabine, leucovorin, and oxaliplatin), or FOLFIRI (5FU, leucovorin, and irinotecan).

\subsection{Optimal Duration of Chemotherapy}

The optimal duration of initial chemotherapy for advanced CC remains unknown. OPTIMOX-1 and OPTIMOX-2 trials were designed to address the question if continued chemotherapy provides better outcomes compared with intermittent therapy with treatment break following the tumor response [53,54]. Although intermittent oxaliplatin therapy was not associated with inferior outcome, complete discontinuation of chemotherapy was associated with inferior PFS and a trend toward inferior median overall survival ( 20 versus 24 months, $p=0.42$ ). Of note in OPTIMOX-2 after attainment of response, disease was allowed to progress back to baseline levels before the reintroduction of chemotherapy. The MRC COIN trial, in which 1630 patients were randomly assigned to continuous therapy versus 3 months of therapy followed by a chemotherapy holiday until disease progression failed to support non-inferiority of intermittent therapy compared with continuous treatment (18 versus 19.6 months) [55].

In general, the decision for treatment breaks must be individualized according to treatment goals, tolerance of and response to therapy, disease burden and location, and presence of symptoms. Because of cumulative neurotoxicity associated with oxaliplatin based therapy intermittent treatment approaches appear to be necessary for most patients.

\subsection{Anti-VEGF Therapy}

\subsubsection{Bevacizumab}

Bevacizumab is a humanized monoclonal antibody directed against vascular endothelial growth factor-A, a member of a family of VEGF-receptor-activating ligands. The magnitude of benefit may differ based on the chemotherapy regimen with which bevacizumab is partnered [56,57]. In a key phase II trial, 813 patients were randomly assigned to bevacizumab versus placebo in combination with an irinotecan containing regimen (IFL). Addition of bevacizumab to IFL was associated with a median OS of 20.3 months compared with 15.6 months in the IFL group. The median PFS was 10.6 months in the group given IFL plus bevacizumab, as compared with 6.2 months in the group that received only IFL; the corresponding rates of response were $44.8 \%$ and $34.8 \%$ respectively $(\mathrm{P}=0.004)$ [22].

On the other hand, modest benefit was noted when bevacizumab was added to oxaliplatin based regimen in chemotherapy naïve patients. In a phase III trial 1401 patients were randomly assigned bevacizumab versus placebo in combination with oxaliplatin based regimens (FOLFOX/XELOX) [58]. Median PFS was 9.4 months in the bevacizumab group and 8.0 months in the placebo group. No significant difference was noted in response rate and overall survival between the two groups. Of note only $29 \%$ and $47 \%$ of bevacizumab and placebo recipeents, respectively, were treated until progression.

Several registry-based retrospective studies have shown a better outcome if bevacizumab continues beyond progression as a first-line bevacizumab-containing regimen. A phase III trial has recently confirmed and revealed that continuation of bevacizumab with a 2 nd line fluoropyrimidine-based chemotherapy regimen was associated with a significant improvement in OS of 11.2 versus 9.8 months [59].

\subsubsection{Aflibercept}

Aflibercept is a recombinant fusion protein that binds to human VEGF-A, VEGF-B, and placental growth factor and inhibits the binding of these ligands to their respecttive receptors. In the placebo-controlled phase III trial, 1226 patients with oxaliplatin-refractory advanced CC were randomly assigned to aflibercept versus placebo in combination with FOLFIRI [29]. The preliminary data revealed a significantly longer median overall survival of 13.5 versus 12.1 months. 


\subsubsection{Regorafenib}

Regorafenib is an orally active inhibitor of angiogenic, stromal, and oncogenic receptor tyrosine kinases. In a phase III CORRECT trial, it has demonstrated activity in advanced chemotherapy-refractory CC [30]. In this study, 760 patients were randomly assigned to best supportive care with regorafenib or placebo. In a preliminary report, patients treated with regorafenib had a modest improvement in median overall survival (6.4 versus 5 months, hazard ratio $0.77,95 \% \mathrm{CI} 0.64-0.94$ ).

\subsection{Anti-EGFR Monoclonal Antibodies}

The efficacy of monoclonal antibodies (mAbs) cetuximab and panitumumab targeting epidermal growth factor receptor (EGFR) alone or in combination with cytotoxic agents have demonstrated efficacy in both chemotherapy-refractory and untreated advanced CC [24-27]. Several clinico-pathological factors have been proposed to predict response to anti-EGFR mAbs (Table 1). Among those predictive factors KRAS gene mutational status has emerged as a robust biomarker. Approximately $30 \%$ to $40 \%$ of colorectal cancers harbor a mutation in the KRAS gene. KRAS mutations predict lack of clinical benefit to cetuximab or panitumumab therapy and patients with a known KRAS mutation should not be treated with cetuximab or panitumumab $[60,61]$.

\subsubsection{Cetuximab or Panitumumab in Chemotherapy Naïve Patients (1st Line Therapy)}

In the CRYSTAL trial, 1198 chemotherapy naïve patients randomly received FOLFIRI with or without

Table 1. Potential predictive molecular biomarkers for response anti-EGFR therapies.

\begin{tabular}{|c|c|}
\hline Relationship to response & Biomarker \\
\hline $\begin{array}{l}\text { Predicts lack of response and } \\
\text { now incorporated into clinical } \\
\text { practice }\end{array}$ & KRAS mutation \\
\hline $\begin{array}{l}\text { Very likely to predict lack of } \\
\text { response }\end{array}$ & $\begin{array}{l}\text { Mutation or lack of expression } \\
\text { of PTEN; mutation of BRAF or } \\
\text { PIK3CA }\end{array}$ \\
\hline \multirow{2}{*}{ May predict lack of response } & $\begin{array}{l}\text { Increased HER2 gene copy } \\
\text { number }\end{array}$ \\
\hline & $\begin{array}{l}\text { Increased EGFR gene copy } \\
\text { number }\end{array}$ \\
\hline $\begin{array}{l}\text { SMay predict increased } \\
\text { likelihood of response }\end{array}$ & $\begin{array}{l}\text { Increased EGFR phosphorylation } \\
\text { Overexpression of altemative } \\
\text { EGFR ligands (amphiregulin } \\
\text { and/or epiregulin) pAKt } \\
\text { overexpression }\end{array}$ \\
\hline Other potential markers & $\begin{array}{l}\text { Markers of angiogenesis and cell } \\
\text { cycle regulation; transcription } \\
\text { factors }(\mathrm{VEGF}, \mathrm{IL}-8, \mathrm{COX}-2, \\
\text { cyclin } \mathrm{D}, \mathrm{NK} \mathrm{B})\end{array}$ \\
\hline
\end{tabular}

cetuximab [26]. Patients with KRAS wild-type tumor had a significantly better median overall survival of 23.5 months when treated with cetuximab in combination with FOLFIRI compared with median OS of 20 months with FOLFIRI alone. The rate of surgery for metastases was higher in the cetuximab-FOLFIRI group than in the FOLFIRI group alone ( $7.0 \%$ vs $3.7 \%)$. In the PRIME trial addition of panitumumab to FOLFOX significantly improved both response rate (55\% vs $48 \%$ ) and median PFS (9.6 versus 8 months) in 656 patients with KRAS wild-type tumor [28]. On the contrary, MRC COIN trial failed to demonstrate survival benefit with the addition of cetuximab to oxaliplatin based therapy (FOLFOX or CAPOX) in patients with KRAS wild-type tumor [62].

The optimal first line biologic therapy in patients with advanced CC remains undecided. Currently, the US Intergroup trial C80405 is addressing this question and is evaluating the role of first line cetuximab versus bevacizumab in combination with either FOLFIRI or FOLFOX in patients with KRAS wild-type tumors [63].

\subsubsection{Cetuximab or Panitumumab Following First Line Chemotherapy (2nd Line Therapy)}

Addition of cetuximab or panitumumab to irinotecanbased chemotherapy following progression on oxaliplatin containing first line chemotherapy has shown greater treatment response. For instance, in the EPIC trial 1300 patients with FOLFOX refractory disease were randomly assigned to single-agent irinotecan with or without cetuximab [64]. The addition of cetuximab was associated with a significant improvement in PFS (4 versus 2.6 months). Similar results were noted with panitumumab. In a phase III trial addition of panitumumab to FOLFIRI was associated with a significant improvement in PFS (5.9 versus 3.9 months) in patients with KRAS wild tumor [65].

\subsubsection{Cetuximab or Panitumumab in Chemotherapy Refractory Patients (3rd Line Therapy)}

Both cetuximab and panitumumab have demonstrated efficacy as third-line therapy among patients with wildtype KRAS CC that is refractory to irinotecan and oxaliplatin $[24,25,27]$. The CO-17 study revealed a median PFS of 3.8 months among cetuximab-treated patients with KRAS-wild type tumor versus 1.9 months among those treated with best supportive care. In addition, the investigators reported a significant improvement in overall survival (9.5 months vs 4.8 months) [25]. Similar results were observed with panitumumab-treated patients with wild type KRAS tumors who had a mean PFS of 12.3 weeks compared to 7.3 weeks with best supportive care alone [27]. As cross-over was permitted, this trial was unable to demonstrate an overall survival advantage.

Evidence suggests that the combination of cetuximab 
and irinotecan is more effective than cetuximab monotherapy. In the randomized BOND study, higher response rates $(22.9 \%$ vs $10.8 \%)$ and a longer PFS (4.1 months vs 1.5 months) were observed in patients treated with a combination of irinotecan than those receiving cetuximab alone [24]. However, it is to be recalled that the BOND study was conducted in an unselected population that included wild- and mutated-type KRAS tumors.

\subsection{Dual Antibody Therapy (Combination of Anti-EGFR and Anti-VEGF mAbs)}

Two randomized phase III (PACCE and the CAIRO2) trials evaluated the role of concurrent use of dual antibodies targeting VEGF and the EGFR in chemotherapy naïve patients $[66,67]$. Unexpectedly, the addition of the anti-EGFR mAbs to bevacizumab containing regimen resulted in a detrimental effect in both studies. In the CAIRO2 study, involving 755 patients, the efficacy of the addition of cetuximab to CAPOX plus bevacizumab was assessed [66]. The PFS was significantly decreased when cetuximab was added, with a median of 9.4 versus 10.7 months, respectively. The median OS was not significantly different.

In the PACCE trial, oxaliplatin-based or irinotecanbased chemotherapy plus bevacizumab with or without panitumumab was studied. In 823 oxaliplatin-treated patients, both the median PFS and OS were significantly worse when panitumumab was added (10.0 versus 11.4 months, and 19.4 versus 24.5 months respective) [67]. Hence, outside the setting of a clinical trial dual antibody therapy is not recommended for routine clinical use.

\subsection{Systemic Therapy for Resectable or Potentially Resectable Metastataic Liver Disease}

The liver is the dominant site of metastases in patients with CC and is involved in approximately $25 \%-30 \%$ of patients. It accounts for at least two thirds of $\mathrm{CC}$ deaths [8]. Over the past decade improvements in outcomes of patients with advanced $\mathrm{CC}$ have been attributed to the availability of novel agents resulting in a growing number of hepatic resections in selected patients with liver metastases. Approximately $20 \%$ to $30 \%$ of patients with liver metastases are potential candidates for surgical resections (Figure 2) [7,8]. Phase III trials have demonstrated improvements in recurrence free survival with the use of peri-operative chemotherapy in patients with surgically resectable disease [69,70]. The EORTC 40983 study compared perioperative chemotherapy with 12 cycles of FOLFOX to surgery alone in patients with resectable liver metastases. The 3-year PFS was signifycantly improved from $28.1 \%$ for the surgery-alone group to $33.2 \%$ for the perioperative FOLFOX group [68].

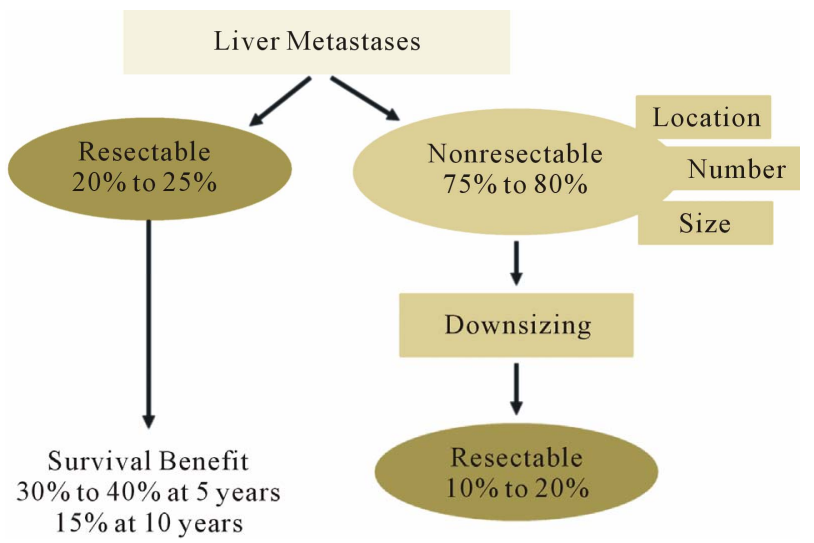

Figure 2. Outcome of patients with liver metastases in colorectal cancer.

Additionally, it has been reported that $\sim 10 \%-20 \%$ (Figure 2) of patients with "initially unresectable" metastatic liver disease can have a substantial response to a combination of chemotherapy and biologic agents - "the so called conversion therapy". Such patients are then able to undergo complete surgical resection of the metastases [70-72]. Five-year survival rates in selected patients following metastasectomy is approximately $40 \%-50 \%$ compared with $10 \%$ using chemotherapy alone $[7,8,70]$.

\section{Part 2: Surgical Treatment}

The past ten years have seen an increasing appreciation for the surgical role in the management of advanced CC. Between $30 \%$ - 38\% of patients diagnosed with stage IV $\mathrm{CC}$ will undergo one or more major surgical procedures [73]. Surgical intervention may be performed with a curative intention or for symptomatic palliation [74]. At initial disease presentation, possibility of resection, whether immediately or with systemic therapy, is of primary concern [2]. It is expected that in the near future, further refinement of surgical procedures will increase the efficacy of such resections [75].

\subsection{Surgical Management of Primary Tumor}

Patients with resectable tumors at presentation have the best outcomes [76]. Patient selection is gradually shifting from the traditional parameters of patient age, presence of metastases, and response to chemotherapy, towards molecular biomarkers [31]. The precise type of resection depends on the primary location of the tumor with the principle objective being to remove the primary tumor along with its lymphovascular supply such as a right hemicolectomy for lesions of the cecum, ascending colon, and hepatic flexure. The length of bowel resection depends on the vessel supplying the affected segment, as lymphatics accompany the main arterial supply and the goal of curative surgical resection is to remove both the 
tumor and its lymphovascular supply [77]. This surgical approach along with metastasectomy is associated with a 5 -year survival rate of $30 \%$ [73]. In the event of recurrence, a second resection combined with chemotherapeutics is indicated [78]. A major breakthrough in the treatment of advanced CC has emerged with the advent of modern chemotherapy. Patients who previously would have been treated palliatively have the potential to achieve long-term survival. Five- and ten-year survival is reported to be $33 \%$ and $22 \%$ with a median survival of 39 months [79].

Asymptomatic stage IV colon cancer presents a therapeutic dilemma. Surgical intervention is not without complications. Statistics including an operative mortality of up to $8 \%$ and post-operative complications in $32 \%$ of patients suggest that surgical intervention may not be indicated in all patients who are asymptomatic. Primary resection, however, has been shown to confer a significantly longer overall survival than patients managed only with chemotherapeutics [80]. A recent study by Anwar et al. suggests that prognostic factors such as tumor burden and performance status, should dictate the necessity for surgical resection rather than the presence of symptoms [9]. Results of a meta-analysis by Stillwell, Buettner and Ho suggest disabling symptoms that arise from the primary tumor as it progresses, including weight loss, pain, nutritional depletion, and anemia/hemorrhage may be prevented by resection of the primary lesion. Additionally, emergency procedures are more commonly associated with complications than elective surgeries; therefore, these authors suggest an improved post-operative course may be associated with early surgical intervention [81, 82]. Additional arguments in support of this position include the possibility for peritoneal exploration to more accurately stage the disease extent and an enhanced response to chemotherapeutics with the potential for curative outcomes due to reduction of tumor burden [81]. Finally, the authors indicate there is a paucity of evidence proving a delay in chemotherapy is associated with reduced survival. The authors conclude that resection of the primary tumor in asymptomatic patients may prolong survival by $\sim 6$ months depending on the extent of hepatic/peritoneal/omental metastases, serum ALP and albumin, and the general health of the patient [81]. These results are disputed in a review by Scheer et al. who conclude that the minimal benefit of resection does not counterbalance the significant morbidity and mortality nor the risk of delaying chemotherapy. The authors suggest asymptomatic patients should be treated with chemotherapy, with resection being deferred to patients who develop complications from the primary tumor such as obstruction or hemorrhage [83].

Despite advances to include more patients into the "curative" resection group, the most common treatment of stage IV CC remains palliative colon resection conducted primarily to prevent complications such as obstruction, perforation and hemorrhage [9,83]. It is suggested that resection of the primary may also confer an overall survival benefit. A multivariate analysis by Kim et al compared palliative treatments and found the longest median survival in patients treated with resection + postoperative chemotherapy (14 months) compared with chemotherapy alone ( 8 months) or resection alone (5 months) [11]. It has, however, been suggested that surgical alteration of the body's immune response may increase tumor growth postoperatively [9]. As these patients are usually more ill than those able to undergo resection with a curative intent, relatively high rates of morbidity $(20 \%-45 \%)$ and mortality $(5 \%-8 \%)$ have been reported [9]. Further research is required to effectively identify patients who will benefit from surgery [9].

We have performed a systematic review with metaanalysis to assess the benefit of primary tumor resection in stage IV CC as presented at the European Society of Medical Oncology 2012 annual meeting. Our review showed that the median survival of the primary tumor resection group was 15.2 months (range: 10 - 30.7) compared with 11.4 months (range: 3 - 22) in the non-resection group. Hazard ratio for survival was 0.69 (95\% CI: $0.61-0.79)$ favoring surgical resection. Mean post-operative mortality and non-fatal complications rates were $4.9 \%$ (95\% CI: 0 - 9.7) and 25.9\% (95\%CI: 20.1 - 31.6) respectively. The mean primary tumor complication rates and non-resection procedure rate in the non-resection group were $29.7 \%$ (95\%CI: 18.5 - 41.0$)$ and $27.6 \%$ (95CI: 15.4 - 39.9) respectively. One can conclude that although the retrospective data favors primary tumor resections in patients with advanced $\mathrm{CC}$, the low quality evidence necessitates the future need of adequately powered well designed prospective randomized trials to address this question [84].

\subsection{Surgical Management of Malignant Bowel Obstruction}

Obstruction of the colonic lumen is a serious complication of colon cancer that is most commonly associated with advanced and metastatic disease. On diagnosis, 10\% $20 \%$ of patients with advanced CC will have a partial bowel obstruction, $8 \%-29 \%$ a complete [11] and $10 \%$ $28 \%$ of all patients with CC will develop a malignant bowel obstruction over the course of their disease [80]. Obstructions may also be secondary to resection postoperatively [85]. Long-term survival in these patients ranges from 4 - 9 months with CT scanning being a critical component of the pre-operative workup [86]. Obstructions are more common in patients with left-sided tumors $(21 \%)$ when compared with right-sided tumors 
(12\%) [83]. Such obstructions have traditionally been treated with surgery involving a two-stage procedure of resection and stoma formation, which has been associated with a high morbidity and mortality [87]. A onestage operation including either a subtotal colectomy + ileocolic anastomosis or a segmental resection + primary anastomosis after intraoperative colonic decompression have been reported; however, this aggressive approach is reported to be achievable in only two-thirds (66\%) of patients [88]. The mortality rate of colostomy is high and may greatly diminish the quality of life, especially as up to $50 \%$ of patients will never have a colostomy reversal [89].

Additional palliative procedures that have been reported for the treatment of malignant bowel obstruction include G-tubes, lysis of adhesions, bypass, and colorectal stents [86]. Techniques such as balloon dilatation and laser ablation have been reported to have low success in treating obstructions [90]. The optimal management for stage IV CC with obstruction remains controversial due to multiple treatment options and conflicting research outcomes often based on nonrandomized trials with a small sample size [90,91]. Nevertheless, appropriate treatment should be chosen by examination of the risks and benefits of each option in the context of each individualized patient's goals and expectations.

\subsection{Non-Surgical Management of Malignant Obstruction}

Since they were first proposed in the early 1990s, the use of Self Expandable Metallic Stents (SEMSs) has remained debatable for the treatment of colonic obstruction due to malignant growth. SEMSs, are tubes made of metal, placed at the level of an obstruction that expand in the presence of normal body temperature due to the elasticity of Nitinol [92]. Stent placement may be performed endoscopically using both fluoroscopic and endoscopic guidance or radiologically using only fluoroscopic guidance [85]. Efficient stent placement is a surgical skill that takes a minimum of 20 cases to be considered "experienced" [90]. Indications for SEMSs are two-fold: they may be used as a palliative measure, eliminating the need for stomas in incurable patients, or as a bridge to surgical treatment, acting to decompress the colon [87]. Any treatment of colonic obstruction requires decompression to prevent colonic necrosis and perforation [85]. Stents can reach this endpoint without the risks associated with surgical trauma in high-risk patients [93]. Multiple studies have confirmed an improved outcome in patients treated with SEMSs as an elective rather than an emergency procedure. A death rate of $15 \%-20 \%$ and a complication rate of approximately $50 \%$ have been reported in association with emergency stent placement [11]. It is therefore recommended that patients presenting with acute obstruction without evidence of perforation should be stabilized with the stent placement performed as an elective/semi-elective surgery [92]. This may also allow time for surgical staging, treatment planning, administration of neoadjuvant therapies and patient consultations [90].

The balance between benefits and challenges of using this noninvasive technique does not strongly promote or discourage their use; therefore, the literature remains at odds on the clinical applications of SEMSs. No difference in survival has been reported between patients treated with stents vs surgery [88]. The long-term efficacy and safety of SEMS has yet to be fully elucidated, complicating treatment comparisons, particularly, as the survival of patients with unresectable $\mathrm{CC}$ has increased from 11 - 13 months to 14.8 - 21.5 months [94]. The less invasive nature of SEMSs does not require a general anesthetic as there is no need for a laparotomy and therefore hospital stays are shorter [87,92]. A study by Karoui et al. found the median hospital stay to be five days shorter for patients treated with SEMSs as opposed to surgery (8.0 vs 13.5 days). In this context, a rapid recovery allowed earlier administration of chemotherapeutics (14.0 vs 28.5 days) [88]. Stents additionally offer the advantage of an improved quality of life with greater comfort as stoma formation may be avoided and is therefore more cost effective than surgery [88,92]. High rates of technical (96\%) and clinical success (92\%) have been achieved; however, this study also reported that nearly one-third of patients $(31 \%)$ required a repeat colonoscopy for symptoms of obstruction [92]. Complications directly associated with stent placement include: stent migration $(5.5 \%-11 \%)$, re-obstruction $(7 \%-12 \%)$, perforation ( $3 \%-5.6 \%)$, bleeding, tenesmus, intractable nausea and abdominal pain $[89,94,95]$. Such complications occur in up to $30 \%$ of patients; however, it must be noted that patients receiving stents are often poor surgical candidates with highly advanced disease [11,89]. Such patient selection may also explain the poor 1- and 2-year survival rates of cases treated with SEMSs compared with surgical resection (16.7\% and $2.8 \%$ vs $44.2 \%$ and $21.27 \%$ ) [91]. When combined with bevacizumab, the risk of perforation increases [85]. Two recent studies found a similar complication rate in patients treated surgically and in those with stents [93,94]. Complications of SEMS use has been reported in patients with low volumes of hepatic replacement by metastases, as these patients risk outliving the stent's viability [87]. Overall, the stent-related death rate remains less than $0.6 \%$ with a median survival of 5 months [11]. In light of these complications, it is recommended that patients should be carefully observed, with generous laxative use, and those who cannot be closely monitored should be treated by 
diverting colostomy $[92,93]$.

\subsection{Surgical Management of Metastases}

Stage IV colon cancer is characterized by the presence of extra-colonic tumours. The most common sites of metastases in descending order of frequency include the liver $(11.4 \%)$, peritoneum $(6.4 \%)$, lung $(1.6 \%)$, bone $(0.3 \%)$ and brain $(0.1 \%)$ [77]. Similar to primary tumor treatment protocols, metastatic lesions must also be handled on an individualized basis. Patients should be categorized based on their resectability. Whenever possible, metastasectomy should be considered as it offers the possibility of a cure. Complete surgical resection is reported to improve survival to $35 \%-60 \%$ in selected patients [4]. Mortality rates associated with resection of metastases are falling with concomitant improvements in median survival [76]. Resection of isolated metastases regardless of their location is associated with a prolonged survival [96]. In the event of recurrence, second metastasectomies are also associated with a low peri-operative mortality [78]. In light of these positive outcomes, the therapeutic goal in patients with borderline resectable tumors is to shift them towards a resectable status using modern chemotherapy and biologic agents [76]. Optimization of these peri-surgical systemic therapies remains a subject requiring further study as previously discussed [74]. Unfortunately, only a subset of patients with initially unresectable metastases have substantial responses with systemic therapy to undergo curative surgery. The majority of the patients thus require alternative treatment strategies.

\subsubsection{Liver}

The liver is the most common site of metastatic disease from colon cancer. One quarter of all patients presenting with colon cancer will have hepatic metastases at initial presentation and over half of all patients with colon cancer will eventually develop these metastases over the course of their disease $[74,83,96]$. Lesions in this organ may be isolated or multiple. Treatment options for these metastases include surgical resection, local ablation therapy, transarterial chemoembolization, radiomicrosphere therapy, isolated hepatic perfusion, and therasphere therapy [2]. Such procedures are associated with a mortality rate $<3 \%$ and may achieve 5 - and 10 -year post-resection survival rates of $38 \%$ and $22 \%$ respectively [31]. Solitary metastases in particular confer an excellent prognosis, with five-year overall survival rates approaching $70 \%$ [76]. Poor prognostic factors include extra-hepatic disease, $4+$ and/or bilateral lesions, and surgical margins $<1$ $\mathrm{cm}$ [96].

The primary treatment plan for these lesions involves resection with the aim of improving long-term survival rates [79]. Traditionally hepatic metastasectomy has been considered a curative treatment in selective patients [2]. Contraindications to hepatic resection include: the inability to completely resect the primary tumor or the hepatic tumor with negative margins, the inability to preserve adequate hepatic tissue, or the presence of precluding medical comorbidities [96]. In the past, the presence of extra-hepatic metastases was a contraindication for resection; however, recent evidence demonstrates that though the survival in this population is lower than in patients with isolated liver metastases, it remains superior compared with systemic treatment alone [74]. The timing of this intervention remains undecided as equivalent outcomes are reported in patients with simultaneous synchronous vs metachronous staged colectomy and hepatectomy. Traditionally, patients were treated with a staged approach; however, it is suggested synchronous surgery is a simpler operation [96]. Resection may involve a lobectomy, an extended lobectomy, a segmentectomy, or a non-anatomic wedge resection. Surgeons must balance the desire to preserve the maximal amount of liver tissue with the importance of a negative margin. There have been no studies comparing tissue preserving techniques vs. a formal anatomic resection. A negative margin has been associated with a greater long-term prognosis, yet it is critical that post surgically $20 \%-25 \%$ of functioning hepatic tissue in patients with an otherwise normal liver, and $40 \%$ in patients with a diseased liver is retained [2]. Remnant liver volume may be increased by portal vein embolization to induce hypertrophy of the contralateral lobe [74]. Ideally, a margin of $1 \mathrm{~cm}$ at resection is recommended to decrease the risk of microscopic positive margins [2]. Improvements in these surgical techniques have led to an acceptable operative mortality of $<5 \%$ [76] and curative treatment is possible [4]. In patients with borderline resectability, neoadjuvant chemotherapy may increase the resectability of tumors in up to $12 \%$ [96]. Neoadjuvant chemotherapeutics additionally decrease the risk of micrometastatic disease, resulting in the possibility of a long-term cure [2].

Unfortunately the majority of patients with liver involvement will be surgically unresectable or the patient will not be fit for surgery. Patients may be deemed "unresectable" in the presence of extrahepatic metastases, tumor proximal to any major vasculature, an insufficient hepatic reserve, or an inadequate general patient performance status [97]. These patients should first be given chemotherapy as up to $12 \%$ may be converted to surgical resectability [96]. Such non-operative procedures offer the advantage of enhanced patient safety, percutaneous treatment options, and increased hepatic parenchymal sparing [2]. In these instances, patients are often treated with hepatic embolization or radiofrequency ablation. Hepatic arterial infusion is an effective salvage therapeu- 
tic option, with response rates of $66 \%$ at 6 months in patients treated with intra-arterial irinotecan and similar results for intra-arterial oxaliplatin [74]. Technical complications inhibit a more widespread use of this treatment modality [74].

Radiofrequency ablation (RFA), cryotherapy, laser photocoagulation, microwave ablation, and focused radiotherapy may be used in isolation for unresectable disease or in combination with surgical resection $[2,74]$. These various techniques have similar outcomes [74]. RFA has evolved as the preferred method over the past 10 - 15 years due to its great efficacy and low complication rate [97]. When used in combination with surgical resection, ablation causes a shift from a borderline resectable tumor to one suitable for surgical intervention. When used to treat unresectable tumors alone, this method is not suggested for: metastases $>4 \mathrm{~cm}$, tumors adjacent to large vessels, the presence of extrahepatic disease or when the bowel is adherent to the liver [97]. Proximity to the portal triads or hepatic veins precludes RFA due to the risk of bile duct injury, hepatic necrosis, or inadequate tumor cell death in this area [2]. Alone, RFA confers a greater risk of recurrence with a shorter time to progression than surgical treatment [74], with local recurrence rates as high as $34 \%$ often associated with size and location of the tumors [2]. Though as a standalone treatment RFA is inferior to resection, 1-year survival rates have been reported as high as $78 \%$ [96]. It is hypothesized that, as this technique develops and improves, there may be a fundamental shift in the treatment of liver metastases from surgical resection to RFA [76]. An alternative treatment for liver metastases is radioembolization with yttrium-90 microspheres, which has been shown to be both safe and efficacious as the majority of the blood to a metastatic tumor is from the hepatic artery rather than the liver parenchyma. This procedure therefore provides targeted high-dose radiation to the tumor with hepatic parenchymal sparing. This procedure is not limited by the size, shape, location, or number of lesions nor patient comorbidities all of which may be limiting factors for surgical resection or RFA [98].

\subsubsection{Lung}

The lungs are a common site for deposition and development of colon metastases, occurring in $20 \%$ of patients during their disease [96]. Lung metastases often signal systemic disease and thus careful examination for other sites of dissemination should be conducted [74].

The first-choice treatment for isolated metastases to the lung remains a pulmonary metastasectomy. Lesions are unfortunately most commonly bilateral and therefore unresectable; however, $1 \%$ - $2 \%$ of patients have disease amenable to surgical intervention [96]. Selection criteria of suitable candidates includes information regarding the number and location of the metastases, the absence of extra-pulmonary disease, an adequate pulmonary function reserve, and the absence of comorbidities that contraindicate surgical intervention [77]. Preoperative CEA levels and the status of the hilar and mediastinal nodes should additionally be examined [31]. Treatment of synchronous primary and pulmonary metastases should initially involve resection of the primary followed by adjuvant radiation and/or chemotherapy, restaging, and finally pulmonary metastasectomy if the disease has remained stable and the tumors are still contained within the lungs. Factors predictive of positive post-metastasectomy outcome include: a lesser number of pulmonary nodules, a disease-free interval $>36$ months between initial diagnosis and metastatic development, and a normal preoperative CEA level [96]. 5-year survival has been reported between $30 \%$ - $60 \%$ with an operative mortality as low as $2 \%$. Recurrences of pulmonary metastasis are high, $>70 \%$. Criteria for secondary resections include: isolation of the recurrence, anatomic resectability and physical ability to tolerate the surgery. The success rates of re-resection are equivalent to the first pulmonary metastasectomy with a 5-year survival over 30\% [96]. Similar to hepatic metastases, patients not suitable for pulmonary resection may benefit from RFA [74].

Combined resection of pulmonary and hepatic metastases is reported to confer long-term benefit, with 5-year survival of $10 \%-43 \%$. Prognostic factors indicative of a better outcome include: disease-free interval of $>1$ year separating recurrences, hepatic isolated metastasis, and patient's age less than 55 years. Physiologically, simultaneous resection is stressful; therefore, a staged approach is normally recommended [96].

\subsubsection{Peritoneal Cytoreductive Surgery}

Peritoneal metastases occur in up to half of the patients with stage IV CC [96] and are associated with significant mortality and morbidity [83]. It is hypothesized that metastases to the peritoneum occur by tumor cell shedding as they pass through the lymphatics or blood stream or via direct extensions. Additionally, epithelial-mesenchymal transition (EMT) is a proposed mechanism that facilitates invasion and metastases in which epithelial cells are changed into dedifferentiated mesenchymal cells resulting in extracellular matrix remodeling [6]. Prior to treatment, a CT scan to determine disease extent is required, as bulky disease along the hepatoduodenal ligament limits the surgical treatment and should instead be treated with systemic chemotherapy [96]. Hyperthermic intraperitoneal chemotherapy (HIPEC) is an effecttive treatment for peritoneal metastasis with the ability to achieve long-term survival [4]. This treatment involves infusion of heated chemotherapeutics, often mitomycin $\mathrm{C}$ or oxaliplatin, directly into the peritoneal cavity during 
cytoreductive surgery [2]. This surgery, including resection of the primary tumor and involved organs, omentectomy, and peritoneal stripping of the carcinomatosis, should be completed prior to HIPEC. This procedure is associated with a high short-term morbidity of $30 \%$ $60 \%$ due to lengthy operative time and extensive intraoperative blood loss [96]. The procedure is associated with a $2 \times$ increased survival benefit compared with control cohorts [31]. The extent of cytoreduction dictates prognosis, with a median survival of 33 months in completeness of cancer resection (CCR)-0 disease (no macroscopic residual disease), 12.5 months with CCR-1 (no residual nodule $>0.5 \mathrm{~cm}$ ), and 8.1 months with CCR-2 (residual bulky carcinomatosis) [96].

\section{Conclusion}

Despite ongoing improved screening techniques, colon cancer remains a leading cause of cancer death in North America, with $20 \%$ - $30 \%$ being diagnosed at an advanced stage. For these patients, systemic chemotherapy with or without targeted biological agents remains the mainstay of management, providing a significant improvement in median and progression-free survival. Surgical resection of metastases is usually recommended with or without adjuvant chemotherapy. In most patients, the goals of treatment are to prolong overall survival while maintaining a good quality of life. In certain patients with metastases limited to the liver, potential cure can be obtained with hepatic metastasectomy. The role of surgery in the management of advanced colon cancer continues to evolve as the efficacy of chemotherapy in decreasing tumor burden increases, thereby increasing the susceptibility of the tumor to resection. Despite these gains, the overall impact of combination regimens in metastatic CC therapy has been relatively modest with improved two-year survivals and no substantive gains in five-year survivals. Further well designed trials using novel approaches are thus warranted. In conclusion, we recommend a multi-modality, team-based approach involving colorectal/hepatobiliary/thoracic surgeons, medi$\mathrm{cal} /$ radiation oncologists, pathologists, interventional radiologists, nurses, and psychosocial support services for continued improved results in the management of metastatic and advanced colon cancer.

\section{REFERENCES}

[1] R. Kanthan, J. L. Senger and S. C. Kanthan, "Fecal Molecular Markers for Colorectal Cancer Screening," Gastroenterology Research and Practice, 2012, Article ID: 184343.

[2] D. L. Bartlettt and E. Chu, "Can Metastatic Colorectal Cancer Be Cured?” Oncology, Vol. 26, No. 3, 2012, pp. 266-275.
[3] Y. N. Hsu, J. K. Lin, W. S. Chen, et al., "A New Classification Scheme For Recurrent or Metastatic Colon Cancer after Liver Metastasectomy," Journal of the Chinese Medical Association, Vol. 74, No. 11, 2011, pp. 493-499. doi:10.1016/j.jcma.2011.09.004

[4] C. Verhoef, J. H. de Wilt, J. W. A. Burger, H. M. W. Verheul and M. Koopman, "Surgery of the Primary in Stage IV Colorectal Cancer with Unresectable Metastases," European Journal of Cancer, Vol. 47, Suppl. 3, 2011, pp. S61-S66. doi:10.1016/S0959-8049(11)70148-4

[5] "What Are the Survival Rates for Colorectal Cancer?" 2012.

http://www.cancer.org/cancer/colonandrectumcancer/deta iledguide/colorectal-cancer-survival-rates

[6] R. Kanthan, J. L. Senger and S. C. Kanthan, "Molecular Events in Primary and Metastatic Colorectal Carcinoma: A Review," Pathology Research International, 2012, Article ID: 597497.

[7] E. K. Abdalla, R. Adam, A. J. Bilchik, et al., "Improving Resectability of Hepatic Colorectal Metastases: Expert Consensus Statement," Annals of Surgical Oncology, Vol. 13, No. 10, 2006, pp. 1271-1280. doi:10.1245/s10434-006-9045-5

[8] V. P. Khatri, N. J. Petrelli and Belghiti, "Extending the Frontiers of Surgical Therapy for Hepatic Colorectal Metastases: Is There a Limit?" Journal of Clinical Oncology, Vol. 23, No. 33, 2005, pp. 8490-8499. doi:10.1200/JCO.2004.00.6155

[9] I. S. Anwar, M. B. Peter, J. Dent and N. A. Scott, "Palliative Excisional Surgery for Primary Colorectal Cancer in Patients with Incurable Metastatic Disease. Is There a Survival Benefit?" Colorectal Disease, Vol. 14, No. 8, 2012, pp. 920-930. doi:10.1111/j.1463-1318.2011.02817.x

[10] K. B. Glimelius and N. Cavalli-Bjorkman, "Metastatic Colorectal Cancer: Current Treatment and Future Options for Improved Survival," Scandinavian Journal of Gastroenterology, Vol. 47, No. 3, 2012, pp. 296-314. doi: $10.3109 / 00365521.2012 .640828$

[11] T. S. K. Kim, C. H. Lee, M. R. Lee and J. H. Kim, "Multivariate Analysis of the Survival Rate for Treatment Modalities in Incurable Stage IV Colorectal Cancer," Journal of the Korean Society of Coloproctology, Vol. 28, No. 1, 2012, pp. 35-41. doi:10.3393/jksc.2012.28.1.35

[12] H. Rosen, G. V. Kornek, et al., "Randomized Comparison of Combination Chemotherapy plus Supportive Care with Supportive Care Alone in Patients with Metastatic Colorectal Cancer," BMJ, Vol. 306, No. 6880, 1993, pp. 752755. doi:10.1136/bmj.306.6880.752

[13] P. M. Hoff, R. Ansari, G. Batist, et al., "Comparison of Oral Capecitabine versus Intravenous Fluorouracil plus Leucovorin as First-Line Treatment in 605 Patients with Metastatic Colorectal Cancer: Results of a Randomized Phase III Study," Journal of Clinical Oncology, Vol. 19, No. 8, 2001, pp. 2282-2292.

[14] P. C. Simmonds, "Palliative Chemotherapy for Advanced Colorectal Cancer: Systematic Review and Meta-Analysis," Colorectal Cancer Collaborative Group, Vol. 321, No. 7260, 2000, pp. 531-535. 
[15] J. Y. Douillard, D. Cunningham, A. D. Roth, et al., "Irinotecan Combined with Fluorouracil Compared with Fluorouracil Alone as First-Line Treatment for Metastatic Colorectal Cancer: A Multicentre Randomised Trial," Lancet, Vol. 355, No. 9209, 2000, pp. 1041-1047. doi:10.1016/S0140-6736(00)02034-1

[16] C. H. Köhne, E. van Cutsem, J. Wils, et al., "Phase III Study of Weekly High-Dose Infusional Fluorouracil plus Folinic Acid with or without Irinotecan in Patients with Metastatic Colorectal Cancer: European Organisation for Research and Treatment of Cancer Gastrointestinal Group Study 40986," Journal of Clinical Oncology, Vol. 23, No. 22, 2005, pp. 4856-4865. doi:10.1200/JCO.2005.05.546

[17] S. Giacchetti, B. Perpoint, R. Zidani, et al., "Phase III Multicenter Randomized Trial of Oxaliplatin Added to Chronomodulated Fluorouracil-Leucovorin as First-Line Treatment of Metastatic Colorectal Cancer," Journal of Clinical Oncology, Vol. 18, No. 1, 2000, pp. 136-147.

[18] C. Tournigand, T. André, E. Achille, et al., "FOLFIRI Followed by FOLFOX6 or the Reverse Sequence in Advanced Colorectal Cancer: A Randomized GERCOR Study," Journal of Clinical Oncology, Vol. 22, No. 2, 2004, pp. 229-237. doi:10.1200/JCO.2004.05.113

[19] G. Colucci, V. Gebbia, G. Paoletti, F. Giuliani, et al., "Phase III Randomized Trial of FOLFIRI versus FOLFOX4 in the Treatment of Advanced Colorectal Cancer: A Multicenter Study of the Gruppo Oncologico Dell'Italia Meridionale," Journal of Clinical Oncology, Vol. 23, No. 22, 2005, pp. 4866-4875. doi:10.1200/JCO.2005.07.113

[20] J. Feliu, A. Salud, P. Escudero, et al., "XELOX (Capecitabine plus Oxaliplatin) as First-Line Treatment for Elderly Patients over 70 Years of Age with Advanced Colorectal Cancer," British Journal of Cancer, Vol. 94, No. 7, 2006, pp. 969-975. doi:10.1038/sj.bjc.6603047

[21] G. Masi, E. Vasile, F. Loupakis, et al., "Randomized Trial of Two Induction Chemotherapy Regimens in Metastatic Colorectal Cancer: An Updated Analysis," Journal of the National Cancer Institute, Vol. 103, No. 1, 2011, p. 21. doi:10.1093/jnci/djq456

[22] H. Hurwitz, L. Fehrenbacher, W. Novotny, et al., "Bevacizumab plus Irinotecan, Fluorouracil, and Leucovorin for Metastatic Colorectal Cancer," New England Journal of Medicine, Vol. 350, No. 23, 2004, pp. 2335-2342. doi:10.1056/NEJMoa032691

[23] C. S. Fuchs, J. Marshall and J. Barrueco, "Randomized, Controlled Trial of Irinotecan plus Infusional, Bolus, or Oral Fluoropyrimidines in First-Line Treatment of Metastatic Colorectal Cancer: Updated Results from the BICC-C Study," Journal of Clinical Oncology, Vol. 26, No. 4, 2008, pp. 689-690. doi:10.1200/JCO.2007.15.5390

[24] D. Cunningham, Y. Humblet, S. Siena, et al., "Cetuximab Monotherapy and Cetuximab plus Irinotecan in Irinotecan-Refractory Metastatic Colorectal Cancer," New England Journal of Medicine, Vol. 351, No. 4, 2004, pp. 337-345. doi:10.1056/NEJMoa033025

[25] D. J. Jonker, C. J. O'Callaghan, C. S. Karapetis, et al., "Cetuximab for the Treatment of Colorectal Cancer," New England Journal of Medicine, Vol. 357, No. 20,
2007, pp. 2040-2048. doi:10.1056/NEJMoa071834

[26] E. Van Cutsem, C. H. Köhne, E. Hitre, J. Zaluski, et al., "Cetuximab and Chemotherapy as Initial Treatment for Metastatic Colorectal Cancer," New England Journal of Medicine, Vol. 360, No. 14, 2009, pp. 1408-1417. doi:10.1056/NEJMoa0805019

[27] E. Van Cutsem, M. Peeters, S. Siena, et al., "Open-Label Phase III Trial of Panitumumab plus Best Supportive Care Compared with Best Supportive Care Alone in Patients with Chemotherapy-Refractory Metastatic Colorectal Cancer," Journal of Clinical Oncology, Vol. 25, No. 13, 2007, pp. 1658-1664. doi:10.1200/JCO.2006.08.1620

[28] J. Y. Douillard, S. Siena, J. Cassidy, et al., "Randomized, Phase III Trial of Panitumumab with Infusional Fluorouracil, Leucovorin, and Oxaliplatin (FOLFOX4) versus FOLFOX4 Alone as First-Line Treatment in Patients with Previously Untreated Metastatic Colorectal Cancer: The PRIME Study," Journal of Clinical Oncology, Vol. 28, No. 31, 2010, pp. 4697-4705. doi:10.1200/JCO.2009.27.4860

[29] E. Van Cutsem, J. Tabernero, R. Lakomy, et al., "Intravenous Aflibercept versus Placebo in Combination with Irinotecan/5-FU (FOLFIRI) for Second-Line Treatment of Metastatic Colorectal Cancer (ADVANCED CC): Results of a Multinational Phase III Trial (EFC10262-VELOUR) (ESMO Abstract O-0024)," Annals of Oncology, Vol. 22, Suppl. 5, 2011, p. v8.

[30] E. Van Cutsem, A. F. Sobrero, S. Siena, et al., "Phase III CORRECT Trial of Regorafenib in Metastatic Colorectal Cancer (Advanced CC)," Journal of Clinical Oncology, Vol. 30., 2012, Abstract 3502.

[31] W. T. C. Chua, W. Liauw, F. Chu and D. L. Morris, "Viewing Metastatic Colorectal Cancer as a Curable Chronic Disease," American Journal of Clinical Oncology, Vol. 34, 2012, pp. 77-80. doi:10.1097/COC.0b013e3181fe4444

[32] N. Petrelli, H. O. Douglass Jr., L. Herrera, et al., "The Modulation of Fluorouracil with Leucovorin in Metastatic Colorectal Carcinoma: A Prospective Randomized Phase III trial. Gastrointestinal Tumor Study Group," Journal of Clinical Oncology, Vol. 7, No. 10, 1989, pp. 1419-1426.

[33] R. M. Goldberg, D. J. Sargent, R. F. Morton, et al., “A Randomized Controlled Trial of Fluorouracil plus Leucovorin, Irinotecan, and Oxaliplatin Combinations in $\mathrm{Pa}-$ tients with Previously Untreated Metastatic Colorectal Cancer," Journal of Clinical Oncology, Vol. 22, No. 1, 2004, pp. 23-30. doi:10.1200/JCO.2004.09.046

[34] M. A. Poon, M. J. O’Connell, C. G. Moertel, et al., "Biochemical Modulation of Fluorouracil: Evidence of Significant Improvement of Survival and Quality of Life in Patients with Advanced Colorectal Carcinoma," Journal of Clinical Oncology, Vol. 7, No. 10, 1989, pp. 1407 1418.

[35] N. Petrelli, L. Herrera, Y. Rustum, et al., "A prospective Randomized Trial of 5-Fluorouracil versus 5-Fluorouracil and High-Dose Leucovorin versus 5-Fluorouracil and Methotrexate in Previously Untreated Patients with Advanced Colorectal Carcinoma," Journal of Clinical Oncology, Vol. 5, No. 10, 1987, pp. 1559-1565. 
[36] A. de Gramont, J. F. Bosset, C. Milan, et al., "Randomized Trial Comparing Monthly Low-Dose Leucovorin and Fluorouracil Bolus with Bimonthly High-Dose Leucovorin and Fluorouracil Bolus plus Continuous Infusion for Advanced Colorectal Cancer: A French Intergroup Study," Journal of Clinical Oncology, Vol. 15, No. 2, 1997, pp. 808-815.

[37] P. M. Hoff, R. Ansari, G. Batist, et al., "Comparison of Oral Capecitabine versus Intravenous Fluorouracil plus Leucovorin as First-Line Treatment in 605 Patients with Metastatic Colorectal Cancer: Results of a Randomized Phase III Study," Journal of Clinical Oncology, Vol. 19, No. 8, 2001, pp. 2282-2292.

[38] E. Van Cutsem, C. Twelves, J. Cassidy, et al., "Oral Capecitabine Compared with Intravenous Fluorouracil plus Leucovorin in Patients with Metastatic Colorectal Cancer: Results of a Large Phase III Study," Journal of Clinical Oncology, Vol. 19, No. 21, 2001, pp. 4097-4106.

[39] D. Cunningham, S. Pyrhönen, R. D. James, et al., "Randomized Trial of Irinotecan plus Supportive Care versus Supportive Care Alone after Fluorouracil Failure for Patients with Metastatic Colorectal Cancer," Lancet, Vol. 352, 1998, pp. 1413-1416. doi:10.1016/S0140-6736(98)02309-5

[40] J. Y. Douillard, D. Cunningham, A. D. Roth, et al., "Irinotecan Combined with Fluorouracil Compared with Fluorouracil Alone as First-Line Treatment for Metastatic Colorectal Cancer: A Multicenter Randomized Trial," Lancet, Vol. 355, No. 9209, 2000, pp. 1041-0147. doi:10.1016/S0140-6736(00)02034-1

[41] L. B. Saltz, J. V. Cox, C. Blanke, et al., "Irinotecan plus Fluorouracil and Leucovorin for Metastatic Colorectal Cancer. Irinotecan Study Group," New England Journal of Medicine, Vol. 343, No. 13, 2000, pp. 905-914. doi:10.1056/NEJM200009283431302

[42] M. L. Rothenberg, A. M. Oza, R. H. Bigelow, et al., "Superiority of Oxaliplatin and Fluorouracil-Leucovorin Compared with Either Therapy Alone in Patients with Progressive Colorectal Cancer after Irinotecan and Fluorouracil-Leucovorin: Interim Results of a Phase III Trial," Journal of Clinical Oncology, Vol. 21, No. 11, 2003, pp. 2059-2069. doi:10.1200/JCO.2003.11.126

[43] A. de Gramont, A. Figer, M. Seymour, et al., "Leucovorin and Fluorouracil with or without Oxaliplatin as First-Line Treatment in Advanced Colorectal Cancer," Journal of Clinical Oncology, Vol. 18, No. 16, 2000, pp. 2938-2947.

[44] A. Grothey, B. Deschler, H. Kroening, et al., "Phase III Study of Bolus 5-Fluorouracil (5-FU)/Folinic Acid (FA) (Mayo) vs Weekly High-Dose 24 h 5-FU Infusion/FA + Oxaliplatin in Advanced Colorectal Cancer (Abstract)" Proceedings of American Society of Clinical Oncology, Vol. 21, 2002, p. 129a.

[45] J. Cassidy, J. Tabernero, C. Twelves, et al., "XELOX (Capecitabine plus Oxaliplatin): Active First-Line Therapy for Patients with Metastatic Colorectal Cancer," Journal of Clinical Oncology, Vol. 22, No. 11, 2004, pp. 2084-2091. doi:10.1200/JCO.2004.11.069

[46] H. T. Arkenau, D. Arnold, J. Cassidy, et al., "Efficacy of
Oxaliplatin plus Capecitabine or Infusional Fluorouracil/ Leucovorin in Patients with Metastatic Colorectal Cancer: A Pooled Analysis of Randomized Trials," Journal of Clinical Oncology, Vol. 26, 2008, p. 5910. doi:10.1200/JCO.2008.16.7759

[47] H. K. Sanoff, D. J. Sargent, M. E. Campbell, et al., "Five-year data and prognostic factor analysis of oxaliplatin and irinotecan combinations for advanced colorectal cancer: N9741" J Clin Oncol, Vol. 26, 2008, pp. 5721. doi:10.1200/JCO.2008.17.7147

[48] A. Falcone, S. Ricci, I. Brunetti, et al., "Phase III Trial of Infusional Fluorouracil, Leucovorin, Oxaliplatin, and Irinotecan (FOLFOXIRI) Compared with Infusional Fluorouracil, Leucovorin, and Irinotecan (FOLFIRI) as First-Line Treatment for Metastatic Colorectal Cancer: The Gruppo Oncologico Nord Ovest," Journal of Clinical Oncology, Vol. 25, No. 13, 2007, pp. 1670-1676. doi:10.1200/JCO.2006.09.0928

[49] J. Souglakos, N. Androulakis, K. Syrigos, et al., "FOLFOXIRI (Folinic Acid, 5-Fluorouracil, Oxaliplatin and Irinotecan) vs FOLFIRI (Folinic Acid, 5-Fluorouracil and Irinotecan) as First-Line Treatment in Metastatic Colorectal Cancer (ADVANCED CC): A Multicenter Randomized Phase III Trial from the Hellenic Oncology Research Group (HORG)," British Journal of Cancer, Vol. 94, 2006, p. 798. doi:10.1038/sj.bjc.6603011

[50] R. M. Goldberg, M. L. Rothenberg, E. Van Cutsem, et al., "The Continuum of Care: A Paradigm for the Management of Metastatic Colorectal Cancer," Oncologist, Vol. 12 , No. 1, 2007, pp. 38-50.

doi:10.1634/theoncologist.12-1-38

[51] M. T. Seymour, T. S. Maughan, J. A. Ledermann, et al., "Different Strategies of Sequential and Combination Chemotherapy for Patients with Poor Prognosis Advanced Colorectal Cancer (MRC FOCUS): A Randomized Controlled Trial," Lancet, Vol. 370, 2007, pp. 143152. doi:10.1016/S0140-6736(07)61087-3

[52] M. Koopman, N. F. Antonini, J. Douma, et al., "Sequential versus Combination Chemotherapy with Capecitabine, Irinotecan, and Oxaliplatin in Advanced Colorectal Cancer (CAIRO): A Phase III Randomized Controlled Trial," Lancet, Vol. 370, 2007, pp. 135-142. doi:10.1016/S0140-6736(07)61086-1

[53] C. Tournigand, A. Cervantes, A. Figer, et al., "OPTIMOX1: A Randomized Study of FOLFOX4 or FOLFOX7 with Oxaliplatin in a Stop-and-Go Fashion in Advanced Colorectal Cancer-A GERCOR Study," Journal of Clinical Oncology, Vol. 24, 2006, pp. 394-400. doi:10.1200/JCO.2005.03.0106

[54] B. Chibaudel, F. Maindrault-Goebel, G. Lledo, et al., "Can Chemotherapy Be Discontinued in Unresectable Metastatic Colorectal Cancer? The GERCOR OPTIMOX2 Studym" Journal of Clinical Oncology, Vol. 27, 2009, pp. 5727-5733. doi:10.1200/JCO.2009.23.4344

[55] R. A. Adams, A. M. Meade, M. T. Seymour, et al., "Intermittent versus Continuous Oxaliplatin and Fluoropyrimidine Combination Chemotherapy for First-Line Treatment of Advanced Colorectal Cancer: Results of the Randomized Phase 3 MRC COIN Trial," Lancet Oncol- 
ogy, Vol. 12, 2011, pp. 642-653. doi:10.1016/S1470-2045(11)70102-4

[56] L. T. Macedo, A. B. da Costa Lima and A. D. Sasse, "Addition of Bevacizumab to First-Line Chemotherapy in Advanced Colorectal Cancer: A Systematic Review and Meta-Analysis, with Emphasis on Chemotherapy Subgroups," BMC Cancer, Vol. 12, No. 1, 2012, p. 89. doi:10.1186/1471-2407-12-89

[57] F. Kabbinavar, H. I. Hurwitz, L. Fehrenbacher, et al., "Phase II, Randomized Trial Comparing Bevacizumab plus Fluorouracil (FU)/Leucovorin (LV) with FU/LV Alone in Patients with Metastatic Colorectal Cancer," Journal of Clinical Oncology, Vol. 21, No. 1, 2003, pp. 60-65. doi:10.1200/JCO.2003.10.066

[58] L. B. Saltz, S. Clarke, E. Díaz-Rubio, et al., "Bevacizumab in Combination with Oxaliplatin-Based Chemotherapy as First-Line Therapy in Metastatic Colorectal Cancer: A Randomized Phase III Study," Journal of Clinical Oncology, Vol. 26, No. 12, 2008, pp. 2013-2019. doi:10.1200/JCO.2007.14.9930

[59] D. Arnold, T. Andre, J. Bennouna, et al., "Bevacizumab (BEV) plus Chemotherapy (CT) Continued Beyond First Progression in Patients with Metastatic Colorectal Cancer (Advanced CC) Previously Treated with BEV plus CT: Results of a Randomized Phase III Intergroup Study (TML Study)," Journal of Clinical Oncolog, Vol. 30, 2012, Abstract CRA3503.

[60] C. S. Karapetis, S. Khambata-Ford, D. J. Jonker, et al., "K-ras Mutations and Benefit from Cetuximab in Advanced Colorectal Cancer," New England Journal of Medicine, Vol. 359, No. 17, 2008, pp. 1757-1765. doi:10.1056/NEJMoa0804385

[61] R. G. Amado, M. Wolf, M. Peeters, et al., "Wild-Type KRAS Is Required for Panitumumab Efficacy in Patients with Metastatic Colorectal Cancer," Journal of Clinical Oncolog, Vol. 26, No. 10, 2008, pp. 1626-1634. doi:10.1200/JCO.2007.14.7116

[62] T. S. Maughan, R. Adams, C. G. Smith, et al., "Identification of Potentially Responsive Subsets When Cetuximab Is Added to Oxaliplatin-Fluoropyrimidine Chemotherapy in First-Line Advanced Colorectal Cancer (aCC): Mature Results of the MRC COIN Trial (Abstract 3502)" Journal of Clinical Oncolog, Vol. 28, 2010, p. 261s.

[63] National Cancer Institute, "Phase III Randomized Study of Cetuximab and/or Bevacizumab in Combination with either Oxaliplatin, Fluorouracil, and Leucovorin Calcium (FOLFOX) or Irinotecan Hydrochloride, Fluorouracil, and Leucovorin Calcium (FOLFIRI) in Patients with Previously Untreated Metastatic Adenocarcinoma of the Colon or Rectum," 2012.

http://www.cancer.gov/search/ResultsClinicalTrialsAdva nced.aspx?protocolsearchid $=5463125$

[64] A. F. Sobrero, J. Maurel, L. Fehrenbacher, et al., "EPIC: Phase III Trial of Cetuximab plus Irinotecan after Fluoropyrimidine and Oxaliplatin Failure in Patients with Metastatic Colorectal Cancer," Journal of Clinical Oncolog, Vol. 26, No. 14, 2008, pp. 2311-2319. doi:10.1200/JCO.2007.13.1193

[65] M. Peeters, T. J. Price, A. Cervantes, et al., "Randomized
Phase III Study of Panitumumab with Fluorouracil, Leucovorin, and Irinotecan (FOLFIRI) Compared with FOLFIRI Alone as Second-Line Treatment in Patients with Metastatic Colorectal Cancer," Journal of Clinical Oncolog, Vol. 28, No. 3, 2010, pp. 4706-4713. doi:10.1200/JCO.2009.27.6055

[66] J. Tol, M. Koopman, A. Cats, et al., "Chemotherapy, Bevacizumab, and Cetuximab in Metastatic Colorectal Cancer," New England Journal of Medicine, Vol. 360, 2009, pp. 563-572. doi:10.1056/NEJMoa0808268

[67] J. R. Hecht, E. Mitchell, T. Chidiac, et al., "A randomized Phase IIIB Trial of Chemotherapy, Bevacizumab, and Panitumumab Compared with Chemotherapy and Bevacizumab Alone for Metastatic Colorectal Cancer," Journal of Clinical Oncolog, Vol. 27, No. 5, 2009, pp. 672-680. doi:10.1200/JCO.2008.19.8135

[68] B. Nordlinger, H. Sorbye, B. Glimelius, et al., "Perioperative Chemotherapy with FOLFOX4 and Surgery versus Surgery Alone for Resectable Liver Metastases from Colorectal Cancer (EORTC Intergroup Trial 40983): A Randomized Controlled Trial," Lancet, Vol. 371, No. 9617, 2008, pp. 1007-1016. doi:10.1016/S0140-6736(08)60455-9

[69] E. Mitry, A. L. Fields and H. Bleiberg, "Adjuvant Chemotherapy after Potentially Curative Resection of Metastases from Colorectal Cancer: A Pooled Analysis of Two Randomized Trials," Journal of Clinical Oncolog, Vol. 26, No. 30, 2008, pp. 4906-4911. doi:10.1200/JCO.2008.17.3781

[70] R. Adam, D. A. Wicherts, R. J. de Haas, et al., "Patients with Initially Unresectable Colorectal Liver Metastases: Is There a Possibility of Cure?" Journal of Clinical Oncolog, Vol. 27, No. 11, 2009, pp. 1829-1835. doi:10.1200/JCO.2008.19.9273

[71] T. Delaunoit, S. R. Alberts, D. J. Sargent, et al., "Chemotherapy Permits Resection of Metastatic Colorectal Cancer: Experience from Intergroup N9741," Annals of Oncology, Vol. 16, No. 3, 2005, pp. 425-429. doi:10.1093/annonc/mdi092

[72] C. Barone, G. Nuzzo, A. Cassano, et al., "Final Analysis of Colorectal Cancer Patients Treated with Irinotecan and 5-Fluorouracil plus Folinic Acid Neoadjuvant Chemotherapy for Unresectable Liver Metastases," British Journal of Cancer, Vol. 97, 2007, pp. 1035-1039. doi:10.1038/sj.bjc. 6603988

[73] Z. Zhao, E. Pelletier, B. Barber, et al., "Major Surgery in Patients with Metastatic Colorectal Cancer in Western Europe," Journal of Gastrointestinal Cancer, Vol. 43, No. 3, 2012, pp. 456-461. doi:10.1007/s12029-011-9349-y

[74] T. C. Chua and D. L. Morris, "Therapeutic Potential of Surgery for Metastatic Colorectal Cancer," Scandinavian Journal of Gastroenterology, Vol. 47, No. 3, 2012, pp. 258-268. doi:10.3109/00365521.2012.640823

[75] N. Brunner and H. J. Nielsen, "Directions for Further Improvements of Treatment of Metastatic Colorectal Cancer," Scandinavian Journal of Gastroenterology, Vol. 47, No. 3, 2012, pp. 363-364. doi: $10.3109 / 00365521.2012 .640837$ 
[76] J. L. Marshall, "Managing Potentially Resectable Metastatic Colon Cancer," Gastrointestinal Cancer Research, Vol. 2, Suppl. 2, 2008, pp. S23-S26.

[77] K. Okuno, "Surgical Treatment for Digestive Cancer," Digestive Surgery, Vol. 24, No. 2, 2007, pp. 108-114. doi:10.1159/000101897

[78] G. Brandi, J. Corbelli and F. de Rosa, "Second Surgery or Chemotherapy for Relapse after Radical Resection of Colorectal Cancer Metastases," Langenbeck's Archives of Surgery, Vol. 397, No. 7, 2012, pp. 1069-1077. doi:10.1007/s00423-012-0974-0

[79] R. Adam, V. Delvart, G. Pascal, et al., "Rescue Surgery for Unresectable Colorectal Liver Metastases Downstaged by Chemotherapy. A Model to Predict Long-Term Survival," Annals of Surgery, Vol. 240, 2004, pp. 644-658.

[80] D. Clements, P. D. Rao, D. Ramanathan, et al., "Management of the Asymptomatic Primary in the Palliative Treatment of Metastatic Colorectal Cancer," Colorectal Disease, Vol. 11, No. 8, 2009, pp. 845-848. doi:10.1111/j.1463-1318.2008.01695.x

[81] A. P. Stillwell, P. G. Buettner and Y. H. Ho, "MetaAnalysis of Survival of Patients with Stage IV Colorectal Cancer Managed with Surgical Resection versus Chemotherapy Alone," World Journal of Surgery, Vol. B4, 2010, pp. 797-807. doi:10.1007/s00268-009-0366-y

[82] S. Stelzner, G. Hellmich, R. Koch and K. Ludwig, "Factors Predicting Survival in Stage IV Colorectal Carcinoma Patients after Palliative Treatment: A Multivariate Analysis," Journal of Surgical Oncology, Vol. 89, No. 4, 2005, pp. 211-217. doi:10.1002/jso.20196

[83] M. G. W. Scheer, C. E. J. Sloots, G. J. van der Wilt and T. J. M. Ruers, "Management of Patients with Asymptomatic Cancer and Synchronous Irresectable Metastases," Annals of Oncology, Vol. 19, No. 11, 2008, pp. 18291835. doi:10.1093/annonc/mdn398

[84] S. Ahmed, R. K. Shahid, A. Leis, et al., "Should Palliative Resection of Primary Tumor Be Performed in Patients with Advanced Colorectal Cancer? A Systematic Review and Meta-Analysis," Annals of Oncology, Vol. 23, Suppl. 9, 2012, Abs531.

[85] E. A. Bonin and T. H. Baron, "Update on the Indications and Use of Colonic Stents," Current Gastroenterology Reports, Vol. 12, No. 5, 2010, pp. 374-382. doi:10.1007/s11894-010-0136-x

[86] K. M. Dalal, M. J. Gollub, T. J. Miner, et al., "Temple, Management of Patients with Malignant Bowel Obstruction and Stage IV Colorectal Cancer," Journal of Palliative Medicine, Vol. 14, No. 7, 2011, pp. 822-928. doi:10.1089/jpm.2010.0506

[87] H. Chouhan, C. X. Wong, P. Maharaj, M. J. Lawrence, A. Hunter and J. W. Moore, "Colorectal Stenting for Malignant Obstruction: An 8-Year Clinical Experience," ANZ Journal of Surgery, Vol. 82, No. 6, 2012, pp. 408-411. doi:10.1111/j.1445-2197.2012.06086.x

[88] M. Karoui, A. Charachon, C. Delbaldo, et al., "Stents for
Palliation of Obstructive Metastatic Colon Cancer," Archives of Surgery, Vol. 142, No. 7, 2007, pp. 619-623. doi:10.1001/archsurg.142.7.619

[89] S. Meisner, F. Gonzalez-Huix, J. G. Vandervoort, A. Repici, et al., "Self-Expanding Metal Stenting for Palliation of Patients with Malignant Colonic Obstruction: Effectiveness and Efficacy on 225 Patients with 12-Month's Follow-Up," Gastroenterology Research and Practice, Vol. 2012, 2012, pp. 296-347. doi:10.1155/2012/296347

[90] D. Williams, R. Law and A. M. Pullyblank, "Colorectal Stenting in Malignant Large Bowel Obstruction: The Learning Curve," International Journal of Surgical Oncology, 2011, Article ID: 917848.

[91] W. S. Lee, J. H. Baek, J. M. Kang, et al., "The Outcome after Stent Placement or Surgery as the Initial Treatment for Obstructive Primary Tumor in Patients with Stage IV Colon Cancer," The American Journal of Surgery, Vol. 203, No. 6, 2012, pp. 715-719. doi:10.1016/j.amjsurg.2011.05.015

[92] A. Lamazza, E. Fiori, A. Schillaci, et al., "Self-Expandable Metallic Stents in Patients with Stage IV Obstructing Colorectal Cancer," World Journal of Surgery, Vol. 36, No. 12, 2012, pp. 2931-2936. doi:10.1007/s00268-012-1767-x

[93] E. Angenete, D. Asplund, M. Bergstrom and P. O. Park, "Stenting for Colorectal Cancer Obstruction Compared to Surgery-A Study of Consecutive Patients in a Single Institution" International Journal of Colorectal Disease, Vol. 27, No. 5, 2012, pp. 665-670. doi:10.1007/s00384-011-1374-6

[94] H. J. Lee, S. P. Hong, J. H. Cheon, T. I. Kim, B. S. Min, N. K. Kim and W. H. Kim, "Long-Term Outcome of Palliative Therapy for Malignant Colorectal Obstruction with Unresectable Metastatic Colorectal Cancers: Endoscopic Stenting versus Surgery," Gastrointestinal Endoscopy, Vol. 73, No. 3, 2011, pp. 535-543. doi:10.1016/i.gie.2010.10.052

[95] M. Mates, D. Dudgeon, L. C. Hookey, et al., "Intractable Nausea in a Patient with Metastatic Colorectal Cancer Following Insertion of a Colonic Stent," Journal of Pain and Symptom Management, Vol. 36, No. 1, 2008, pp. e6e10. doi:10.1016/j.jpainsymman.2008.02.003

[96] N. Mahmound and K. B. Dunn, "Metastasectomy for Stage IV Colorectal Cancer," Diseases of the Colon \& Rectum, Vol. 53, No. 7, 2010, pp. 1080-1092. doi:10.1007/DCR.0b013e3181dcadbc

[97] A. R. Knudsen, A. S. Kannerup, F. V. Mortensen and D. T. Nielsen, "Radiofrequency Ablation of Colorectal Metastases Downstaged by Chemotherapy," ACTA Radiology, Vol. 50, No. 7, 2009, pp. 716-721. doi:10.1080/02841850902991634

[98] M. F. Mulcahy, R. J. Lewandowski, S. M. Ibrahim, et al., "Radioembolization of Colorectal Hepatic Metastases Using Yttrium-90 Micropheres," Cancer, Vol. 115, 2009, pp. 1849-1858. doi: $10.1002 / \mathrm{cncr} .24224$ 